\title{
Minimally Invasive Ivor-Lewis Esophagectomy for Esophageal Cancer After Gastric Bypass
}

\author{
Michael Latzko, MD, FACS ${ }^{1}$ (1) , Bestoun Ahmed, MD, FACS ${ }^{2}$, and Ziad Awad, MD, FACS ${ }^{3}$ \\ ${ }^{1}$ Department of Surgery, Advent Health Sebring, Sebring, FL ; ${ }^{2}$ Department of Surgery, University of Pittsburgh Magee- \\ Women's Hospital Medicine, Pittsburgh, PA; ${ }^{3}$ Department of Surgery, University of Florida College of Medicine, \\ Jacksonville, FL
}

\begin{abstract}
The increasing prevalence of morbid obesity in the United States has been accompanied by a concomitant rise in bariatric surgery to help combat the epidemic. The relationship between obesity and certain cancers, such as esophageal adenocarcinoma, is well established. The need for minimally invasive techniques to treat esophageal cancer in patients with previous bariatric surgery is growing and can present a unique surgical challenge. This report presents the case of a 55-year-old woman with a previous Roux-en-Y gastric bypass who was shown by endoscopy to have an invasive adenocarcinoma located in the distal thoracic esophagus. This necessitated an excision of the thoracic esophagus and the gastric pouch. A laparoscopic and thoracoscopic Ivor-Lewis esophagogastrectomy was performed for this complex patient with esophageal adenocarcinoma. The remnant stomach was fashioned into a gastric conduit using a $60-\mathrm{mm}$ linear stapler with a staple height of $4.1 \mathrm{~mm}$
\end{abstract}

(Echelon, Ethicon Endosurgery, Blue Ash, OH). The reconstruction was performed using a $25-\mathrm{mm}$ Orvil (Covidien, Minneapolis, MN, USA) and EEA 25-mm DST XL (Covidien) to create a circular stapled thoracic esophagogastric anastomosis. A feeding jejunostomy was placed in the residual $130-\mathrm{cm}$ Roux limb. The study demonstrated that minimally invasive esophagectomy is safe and technically feasible with appropriate oncologic outcomes for patients with previous gastric bypass. This cohort of patients will undoubtedly continue to grow in the coming years.

DISCLOSURE There are no conflicts of interest.

Publisher's Note Springer Nature remains neutral with regard to jurisdictional claims in published maps and institutional affiliations.
Electronic supplementary material The online version of this article (https://doi.org/10.1245/s10434-020-08646-9) contains supplementary material, which is available to authorized users.
(C) Society of Surgical Oncology 2020

First Received: 29 February 2020;

Published Online: 2 June 2020

M. Latzko, MD, FACS

e-mail: michael.latzko@adventhealth.com 